\title{
OA04.04. Changes in physiological and psychological markers of stress in hospital personnel after a low-dose mindfulness-based worksite intervention
}

\author{
M Klatt*, B Steinberg, D Marks, A Duchemin \\ From International Research Congress on Integrative Medicine and Health 2012 \\ Portland, Oregon, USA. 15-18 May 2012
}

\section{Purpose}

To determine the efficacy of a pragmatic low dose mindfulness-based worksite intervention on biological and behavioral indices of stress in healthcare professionals caring for seriously ill patients.

\section{Methods}

Participants $(n=32)$ were recruited among the Surgical Intensive Care Unit (SICU) personnel of a large university hospital, and were randomized to intervention or wait-list control groups, stratified by gender. The low dose 8-week mindfulness-based intervention was delivered on site, one hour before shift change.

\section{Results}

Participants were representative of the SICU staff with $69 \%$ nurses, $88 \%$ females, age average of 44 , and 11.8 $( \pm 10.1, \mathrm{SD})$ average years working in the SICU. Participant biological indices of stress, measured by the level of salivary $\alpha$-amylase, was significantly reduced in the intervention group ( $\mathrm{t}=2.562, \mathrm{p}=0.026)$ only. Behaviorally, they rated their experience of stress using the Depression, Anxiety and Stress Scale (DASS-21), and rated sleep over the past month using the Pittsburg Sleep Quality Index (PSQI). There was a significant decrease of the scores on the DASS-21 stress subscale $(t=2.245$, $\mathrm{p}=0.040)$ and a significant improvement in the overall quality of sleep $(\mathrm{t}=2.482, \mathrm{p}=0.027)$ between pre and post assessments in the intervention group with no changes for the wait list group. Work satisfaction also increased significantly $(\mathrm{t}=-3.2020, \mathrm{p}=0.006)$ for the intervention group only.

\section{Conclusion}

These data indicate the effectiveness of a mindfulnessbased intervention delivered at the worksite towards stress reduction for staff in a high stress, hospital environment. The SICU personnel care for trauma 1 and 2 level patients and patients with severe pathology recovering from major surgery, and are confronted with catastrophic events on a regular basis. Given the nature of the job, work-related stressful events in the SICU will not change, but the resiliency tools offered via the intervention may help maintain wellness and prevent the deleterious effects of stress.

Published: 12 June 2012

\section{doi:10.1186/1472-6882-12-S1-016}

Cite this article as: Klatt et al: OA04.04. Changes in physiological and psychological markers of stress in hospital personnel after a low-dose mindfulness-based worksite intervention. BMC Complementary and Alternative Medicine 2012 12(Suppl 1):016.

The Ohio State University College of Medicine, Columbus, USA 